\title{
The PHONOLOGICAL PROCESS WITH TWO PATTERNS OF SIMPLIFIED CHINESE CHARACTERS
}

\section{ZHENG JIN}

Institute of Educational Science, Zhengzhou Normal University, China

zhjin@ucdavis.edu

\section{JUNEHEE LEE}

Department of psychology, Gyeongsang National University, Korea

\section{YANG LEE ${ }^{I}$}

Department of psychology, Gyeongsang National University, Korea

yangleepsy@yahoo.co.kr

\begin{abstract}
This paper analyzed word recognition in two patterns of Chinese characters, cross referenced with word frequency. The patterns were defined as uni-part (semantic radical/component only) and bi-part (including the phonetic radical/component and the semantic radical/component) characters. The interactions of semantic and phonological access in both patterns were inspected. It was observed that in the naming task and the pronunciation-matching task, the subject performance involving the uni-part characters showed longer RT than the bi-part characters. However, with the lexical decision and meaning-matching tasks the uni-part characters showed shorter RT than the bi-part characters. It was also observed that the frequency, which is regarded as a lexical variable, displayed a strong influence. This suggests that Chinese characters require lexical access in all tasks. This study also suggested that the phonological process is primary in visual word recognition; as there is a significant phonological effect in processing the Chinese bi-part characters, resulting in either the facilitation or inhibition of phonology due to the differing demands of the two tasks.
\end{abstract}

Keywords: phonological process, lexical access, word frequency, Chinese character, bilingual

\footnotetext{
${ }^{1}$ The first author and the last author contributed equally to this work. The authors wish to thank reviewers for their comments on a draft of this article.
} 


\section{Introduction}

How people achieve the recognition of letters or words by activating the abstract semantic and phonological codes has been a the subject of psychological research. The Chinese character system was designed in way that was hypothesized to only utilize logographic features (Wang et al. 2000). A Chinese character's "semantically significant radical" works directly towards visual recognition. However, recent studies with Chinese characters point out that a Chinese character may have some functional relationaship with phonology (Hsieh 2006). One type of Chinese character has the phonological radical conjoined with semantic radical. The following are examples of the two types of character as uni-part and bi-part.
(1) uni-part 禾/hé/(grain)
中/zhòng/(hit)
(2) bi-part 禾/hé/(grain) $)_{\mathrm{a}}+$ 中/zhòng/(hit) $)_{\mathrm{b}}=$ 种/zhòng/(plant) $)_{\mathrm{ab}}$

Many chinese characters have a radical component showing its meaning a and another component showing its pronunciation ${ }_{b}$. Radicals are often emphasised,but phonetic components considerably less so.

Based on the particular characteristics of Chinese characters, further studies were conducted concerning semantic and phonological access as postulated in the dual route model (which defines the relation between phonology and semantics) in relation to the influence of word frequency.

\subsection{The Dual Route Model and Cascaded Model}

The Dual Route Model postulated two routes of lexical access and phonological mediation to process letters and words (Baron and Strawson 1976; Coltheart 1985; Coslett 1991; Patterson and Morton 1985). The former operates analogously to a direct route between spelling and meaning (lexical entries). This process is a set of word-specific rules that associates orthographic shapes with lexical entries withouth further decomposition. The latter maps from spelling to pronunciation, in turn to map to meaning (lexical entries).

Coltheart et al (1993) revised the Dual Route Mode and demonstrated a Dual Route Cascaded Model (DRC). The DRC refined the Dual Route Model through two core traits. First, processing throughout the routes is cascaded; that is, any activation in earlier modules immediately flows to later modules before processing in the preceding route completes. Second, there are two routes as the lexical route and the non lexical route. 
Through the lexical route, written language processing is accomplished by three unique but interactive procedures in the following ways: the semantic coding process, the orthographical process, and the phonological process (where "phonology" is roughly equivalent to "pronunciation"). In terms of the direct coding model, printed characters are identified by the semantic coding process with help of the orthographical process, without the phonological process. Conversely, in the phonological coding model, words are accessed through the phonological codes with the orthographical process; then the phonological processing plays a leading role in identification. Finally, the rule based grapheme-phoneme correspondence (GPC) system comes into action to process the written characters (Coltheart et al. 2001). The direct coding model and the phonological coding model are part of the lexical route, while the GPC system belongs to the non-lexical route.

There is no unit in the Chinese writing system that encodes single phonemes, and therefore grapheme-phoneme mappings are impossible in the Chinese orthographic inventory (Tan and Perfetti 1998). Therefore, it is not necessary to discuss the non-lexical route of Chinese word recognition. The two specific types of access, which are called semantic access and phonological access, are divided by the lexical route.

\subsection{The Primacy and the Phonological Code}

The written Chinese character is similar to other logographic representations in that it has no GPC (grapheme-to-phoneme) system so that phonological processing does not occur in visual recognition of Chinese characters or that the character meaning is activated earlier than the phonological representation (Baron and Strawson 1976). In contrast, it was proposed that, under certain conditions, phonology is activated pre-semantically to identify Chinese characters (identification-with-phonology hypothesis). The hypothesis has been examined in several studies (e.g., Perfetti and Tan 1998a,b; Tan and Perfetti 1997a; Tan, Hoosain and Siok 1996; see also Hung, Tzeng and Tzeng 1992; Perfetti and Zhang 1991).

There are several studies (e.g., Strain, Patterson and Seidenberg 1995; Neely 1991) implicating that the phonological codes, the corresponding orthographical representations, and their associated lexical processing are reciprocal to each other. The parallel-access model assumes that two pathways are used in accessing lexical semantics: one from orthography proceeding directly to meaning, and the other from orthography to meaning by phonological mediation (e.g., Xu, Pollatsek and Potter 1999).

It has been raised as a key question as to whether phonology is activated automatically, even pre-semantically. The automatic phonological activation in the recognition of Chinese characters, as proposed by Perfetti and Zhang (1991), suggested that all words in all writing systems were recognized only after verification of a phonological code. However, this is true only in sense that the phonological processing 
occurs very rapidly during the identification of printed words, resulting in automatic phonological activation. This suggestion was supported by Phonological Coherence Hypothesis (Lukatela, Frost and Turvey 1999; Van Orden and Goldinger, 1994) and the Universal Phonological Principle (Perfetti and Zhang 1995).

All of the preceding hypotheses that deal with phonological mediation have been proposed for varieties of characters. The Chinese characters, in which the script-meaning relationship is normally regarded as primarily semantic, could be examined with these hypotheses of phonological mediation. In this study, which tests the above hypotheses, the recognition of Chinese characters was compared in both uni-part and bi-part characters. The former has only semantic radicals. The latter is constructed with the addition of phonetic radicals, which may activate the phonological code in the process of recognition. It suggested that the phonological process is primary in visual word recognition; as there is a significant phonological effect in processing the Chinese bi-part characters, resulting in either the facilitation or inhibition of phonology due to the differing demands of the tasks.

This study investigated word recognition in two kinds of Chinese characters: uni-part (semantic radical/component only) and bi-part characters (including the phonetic radical/component and the semantic radical/component). The subjects were asked to perform two kinds of semantic tasks (lexical decision task and meaning-matching task) or two kinds of phonological tasks (naming task and pronunciation-matching task). The results revealed that, for the semantic tasks, the bi-part characters were responded to more slowly and with less accuracy than uni-part characters; for the phonological tasks, the bi-part characters were responded to faster and with more accuracy than uni-part characters. In addition, word frequency effect was observed regardless of the kinds of characters and tasks. Those results suggested that both phonological activation and lexical access are inevitable when processing Chinese characters.

\section{Method}

This study concluded that the lexically guided process, inherited with the lexical decision tasks and the meaning-matching tasks, was inhibited in the bi-part characters with phonological features, as compared to the uni-part characters without phonological features. In contrast, the phonologically guided process, inherited with naming tasks and pronunciation-matching tasks, was facilitated in the tasks using bi-part characters with phonological features, as compared to the uni-part characters without phonological features. 


\subsection{Purpose of experiment}

This study analyzed four tasks: two varieties of semantic tasks, the lexical decision task and the meaning-matching task; and two varieties of the phonological tasks, the naming task and the pronunciation-matching task. Each task is dominated by one of two processing routes. The lexical decision task and the meaning-matching task involve semantic routes more and the phonological routes less. Conversely, the naming task and the pronunciation-matching task engage the phonological route more and the semantic route less. To find any facilitation or inhibition of each route, this study compared the performances of tasks with uni-part and bi-part characters. When the facilitation or inhibition presents, the RT difference between uni-part and bi-part characters could be observed (Table 1). To find any interaction between the two routes, this study manipulated word frequency as a lexical variable. When the lexical route is activated, the word frequency effect could be observed.

\begin{tabular}{|c|c|c|c|}
\hline & $\begin{array}{ll}\text { Lexical } & \text { decision } \\
\text { /Naming Task } & \end{array}$ & Meaning-matching tasks & Pronunciation-matching task \\
\hline$A$ & $\begin{array}{l}\text { 馆/guăn/( restaurant) } \\
\text { 植/zhí/(plant) }\end{array}$ & $\begin{array}{l}\text { 植 /zhí/(plant)- 种 } \\
\text { /zhòng/(plant) }\end{array}$ & $\begin{array}{l}\text { 众 /zhòng/(crowd)- } \\
\text { /zhòng/(plant) }\end{array}$ \\
\hline$B$ & $\begin{array}{l}\text { 甲/jiă/(shell) } \\
\text { 生/shēng/(grow) }\end{array}$ & $\begin{array}{l}\text { 壳 /ké/(shell)- } \\
/ \text { jiă/(shell) }\end{array}$ & 假/jiǎ/(fake)-甲/jiă/(shell) \\
\hline
\end{tabular}

Table 1: Task interpretation (Rt difference between $A$ and $B$ )

\subsection{Participants}

80 Chinese undergraduates participated in the experiment. They were all native Chinese speakers.

\subsection{Stimuli}

This study sampled a set of 80 legitimate characters, which was composed of 40 bi-part characters and 40 uni-part characters, divided by their frequency levels. The Bi-part character has the phonemic radical to activate the phonological route, compared to uni-part character which has only semantic radical. There was no significant difference 
between the numbers of strokes in each character. The character frequency was calculated by consulting the Modern Chinese character Frequency List ${ }^{2}$. Over 258 millions of characters are identified from the collection of Chinese e-texts. It counted the frequency of 9,933 simplified characters in total 193,504,018 characters. This study defined the low frequency as lower than $0.0159 \%$ and high frequency as above this. This study has four subsets of stimuli; two character patterns by two frequency levels. Each subset has 20 characters.

To apply the lexical decision task, 80 non-characters were invented as arbitrary characters which have no corresponding pronunciation or meaning. So, the lexical decision task had 160 stimuli including 80 non-characters and 80 legitimate characters. The set of non-characters were exempted from the naming task in which only 80 legitimately pronounceable characters were stimulated.

The matching tasks for meaning and pronunciation had the 80 legitimate characters paired correspondingly with 80 related prime characters. In the meaning-matching task the set of 160 targets was divided into 80 meaning-related targets (80 legitimate characters) and 80 unrelated. In the pronunciation-matching task the set of 160 targets was divided into 80 pronunciation-related targets (80 legitimate characters) and 80 unrelated. Each of two matching tasks had 2 lists of stimuli for counterbalance-control. A prime character should be paired not only with a related target but also an unrelated target for comparison-control. While this control could make a repetition bias, the problems was resolved by the between-subject control; if a prime character with a related or unrelated target was treated to a subject, the same prime character with a different target was treated to a different subject. For 2 lists, 160 pairs of prime and target were sampled in each of matching tasks as the meaning-match and the pronunciation-matching task.

\subsection{Design of task}

This study analyzed subjects' performance in four tasks; the lexical decision task stimulated characters and non-characters, and required a response of "character" or "non-character" to each stimulus. The stimuli of the naming task consisted only of legitimate characters; responses were the name of each stimulus. The stimuli of the meaning-matching task were character pairs that were either semantically related or unrelated; responses were whether they were related or unrelated. The stimuli of the pronunciation-matching task were character pairs that were phonologically related or unrelated; responses were whether they were related or unrelated. The task variables were treated as between-subjects variables. The two lists in both matching tasks were also treated as between-subjects variables. The focused variable as character patterns

\footnotetext{
${ }^{2} \mathrm{Da}$, J. Chinese text computing.(2004). http://lingua.mtsu.edu/ chinese-computing
} 
(uni-part and bi-part) and frequency (high and low) were treated as within-subject variables.

\subsection{Procedure}

Character strings were presented one at a time on a PC monitor, subtending below $2^{\circ}$ of visual (characters fit inside a $1 \times 0.4 \mathrm{~cm}$ rectangular space and the distance between the monitor and the subject was $35 \mathrm{~cm}$ ). The presentation of stimuli was controlled by experiment software (E-prime). The instructions were displayed on the monitor and followed after a delay of $1 \mathrm{~min}$ by the practice session and the experimental session. The practice session consisted of 12 trials. Following an interval of $1 \mathrm{~min}$, the experimental session began with the same procedure.

The trial procedure began with the fixation circle appearing on the screen for $500 \mathrm{~ms}$. This was followed by the target event that remained on the screen for $400 \mathrm{~ms}$, requiring subject's perception and response. The subject's response was followed by the mask which was composed of a string of circles for $500 \mathrm{~ms}$, and then a blank screen for $400 \mathrm{~ms}$ before the next stimulus appeared. Each response was checked as right or wrong, and the reaction time in each trial was measured.

The above procedure is standard for four tasks of this study, each of which has a variation of stimuli and response situations. For the lexical decision task, subjects judged each stimulus as a character and non-character, designated with one PC key marked as "character" and the other key as "non-character". The responses for the naming task was input through a microphone attached to a PC, and required the subject to name each target. The meaning-matching task had the stimulation of prime and target; the prime after the fixation was displayed for $400 \mathrm{~ms}$, and was followed by the target which appeared for $400 \mathrm{~ms}$. Subjects pressed one of the keys as "same" and "different", identifying the meaning relation between prime and target. In the pronunciation-matching task, which had the same stimulus event as the meaning-matching task, subjects also judged each pair of prime and target as "same" and "different" regarding their phonological relation.

\section{Results}

The variance (ANOVA) on Reaction Time and Accuracy was analyzed in each of the four tasks; lexical decision, naming, meaning-matching, pronunciation-matching. Two lists to control duplication within subject were treated as a between-subject variable, and Character patterns (bi-part or uni-part) and frequency (high or low) were analyzed as within-subject variables. 


\subsection{Lexical decision and meaning-matching tasks}

The primary expectation of lexical decision and meaning-matching task was that bi-part character would be responded to more slowly and/or with less accuracy than uni-part character. Low frequency character would be responded to more slowly and/or with less accuracy than high frequency character.

In the lexical decision task, the mean latency for bi-part and uni-part characters are $521.77 \mathrm{~ms}(529.60 \mathrm{~ms}$ by items) and $492.41 \mathrm{~ms}$ ( $497.03 \mathrm{~ms}$ by items), respectively, and for low and high frequency characters are $534.62 \mathrm{~ms}(546.40 \mathrm{~ms}$ by items) and $479.56 \mathrm{~ms}$ ( $480.23 \mathrm{~ms}$ by items), respectively. The latency difference of character patterns is significant by subjects $F 1$ and items $F 2, F 1(1,19)=29.16, p<.001, F 2(1,76)=8.78, p<$ .005 . The latency difference of frequencies is significant by subjects $F 1$ and items $F 2, F 1$ $(1,19)=87.37, p<.001, F 2(1,76)=36.29, p<.001$. Mean errors for the bi-part and uni-part characters are $9.9 \%$ and $5.7 \%$, respectively, and for low and high frequency characters are $12.9 \%$ and $2.7 \%$, respectively. The error analysis showed significance for character patterns by subjects as $F 1$, and by items as $F 2, F 1(1,19)=6.77, p<.05, F 2(1$, $76)=4.65, p<.05$. However, the error trend was the reverse of the error-RT trade-off (Fig 1). The error analysis showed significance for frequencies by subject as $F 1$, and by items as $F 2, F 1(1,19)=53.32, p<.001, F 2(1,76)=27.06, p<.001$. However, the error trend was the reverse of the error-RT trade-off (Fig 2).

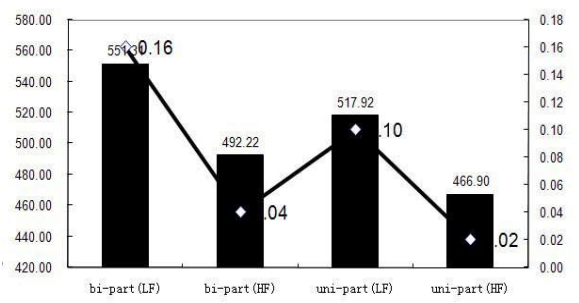

Fig 1. Latency and Error by subject (Lexical decision Task)

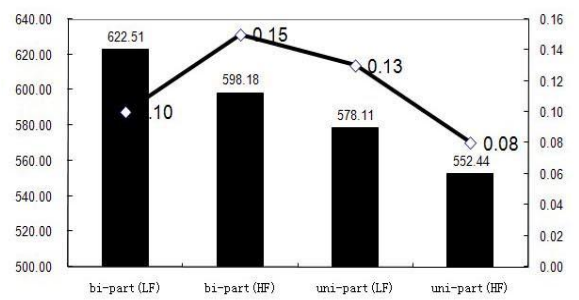

Fig 3. Latency and Error by subject (Meaning-matching Task)

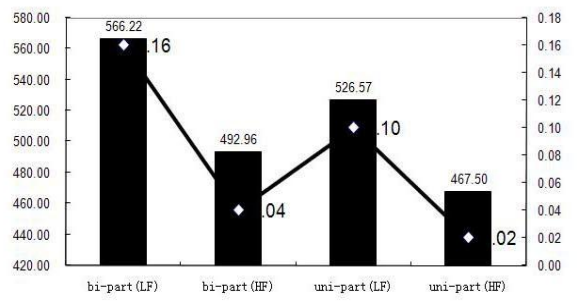

Fig 2. Latency and Error by item (Lexical decision Task)

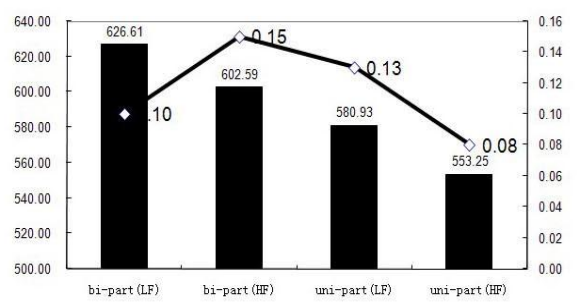

Fig 4. Latency and Error by item (Meaning-matching Task)

In the meaning-matching task, the mean latency for bi-part and uni-part characters are $610.34 \mathrm{~ms}(614.60 \mathrm{~ms}$ by items) and $565.27 \mathrm{~ms}(567.10 \mathrm{~ms}$ by items), respectively, and for 
low and high frequency characters are $600.31 \mathrm{~ms}(603.77 \mathrm{~ms}$ by items) and $575.31 \mathrm{~ms}$ (577.92ms by items), respectively. The latency difference of character patterns is significant by subjects $F 1$ and items $F 2, F 1(1,18)=8.86, p<.01, F 2(1,72)=15.21, p<$ .001 . The latency difference of frequencies is significant by subjects $F 1$ and items $F 2, F 1$ $(1,18)=8.07, p<.05, F 2(1,72)=4.51, p<.05$. Mean errors for bi-part and uni-part characters are $12 \%$ and $10.5 \%$, respectively, and for low and high frequency characters are $11.3 \%$ and $11.3 \%$, respectively. The error analysis showed significance for interaction of character patterns and frequencies by subjects as $F 1, F 1(1,18)=7.79, p<$ .005. However, the error trend was the reverse of the error-RT trade-off (Fig 3, 4)

The review of the above results showed that the lexical decision task and the meaning-matching task converged at the same latency and error pattern as predicted. So, the two tasks worked qualitatively in the same fashion for semantic processing of Chinese characters.

\subsection{Naming, pronunciation-matching task}

The primary expectation of naming and pronunciation-matching task was that bi-part character would be responded to more fast and/or with more accuracy than uni-part character. Low frequency character would be responded to more slowly and/or with less accuracy than high frequency character.

In the naming task, the mean latency for bi-part and uni-part characters are $497.90 \mathrm{~ms}$ ( $496.31 \mathrm{~ms}$ by items) and $538.51 \mathrm{~ms}(548.53 \mathrm{~ms}$ by items), respectively, and for low and high frequency characters are $539.80 \mathrm{~ms}(546.10 \mathrm{~ms}$ by items) and $496.62 \mathrm{~ms}(498.75 \mathrm{~ms}$ by items), respectively. The latency difference of character patterns is significant by subjects $F 1$ and items $F 2, F 1(1,9)=7.18, p<.05, F 2(1,76)=11.82, p<.005$. The latency difference of frequencies is significant by subjects $F 1$ and items $F 2, F 1(1,9)$ $=39.93, p<.001, F 2(1,76)=9.72, p<.005$. Mean errors for bi-part and uni-part characters are $6.5 \%$ and $11.0 \%$, respectively, and for low and high frequency characters are $12.5 \%$ and $5.0 \%$, respectively. The error analysis showed significance for character patterns by subjects as $F 1, F 1(1,9)=6.38, p<.05$. However, the error trend was the reverse of the error-RT trade-off (Fig 5). The error analysis showed significance for frequencies by subjects as $F 1$, and by items as $F 2, F 1(1,9)=22.23, p<.005, F 2(1,76)$ $=7.99, p<.01$. However, the error trend was the reverse of the error-RT trade-off (Fig 6).

In the pronunciation-matching task, the mean latency for bi-part and uni-part characters are $532.45 \mathrm{~ms}(535.68 \mathrm{~ms}$ by items) and $554.20 \mathrm{~ms}(565.16 \mathrm{~ms}$ by items), respectively, and for low and high frequency characters are $564.44 \mathrm{~ms}(576.76 \mathrm{~ms}$ by items) and $522.21 \mathrm{~ms}(524.08 \mathrm{~ms}$ by items), respectively. The latency difference of character patterns is significant by subjects $F 1$ and items $F 2, F 1(1,28)=4.35, p<.05$, $F 2(1,72)=4.16, p<.05$. The latency difference of frequencies is significant by subjects $F 1$ and items $F 2, F 1(1,28)=24.51, p<.001, F 2(1,72)=13.28, p<.005$. Mean errors 
for bi-part and uni-part characters are $4.2 \%$ and $7.2 \%$, respectively, and for low and high frequency characters are $7.8 \%$ and $3.5 \%$, respectively. The error analysis showed significance for character patterns by subjects as $F 1, F 1(1,28)=4.99, p<.05$. However, the error trend was the reverse of the error-RT trade-off (Fig 7). The error analysis showed significance for frequencies by subjects as $F 1, F 1(1,28)=10.13, p<.005$. However, the error trend was the reverse of the error-RT trade-off (Fig 8).

The review of the above results showed that the naming task and the pronunciation-matching task converged at the same latency and error pattern as predicted. So, the two tasks worked qualitatively in the same fashion for phonological processing of Chinese characters.

\section{Discussion}

Every Chinese character can be regarded as a "visual block" which is a syllable as the morpheme, but not as the phoneme. The block can have two patterns: the uni-part or the bi-part. The uni-part is the basic orthographic unit, whereas the bi-part is composed of the semantic radicals and the phonetic radicals, and each radical is regarded as a uni-part character. However, Chinese characters which do not have a phonetic radical are never just "uni-part": a majority of them have two or more than two parts which are perceptually distinguishable (e.g.竹, a character used in this research as a "uni-part" character). Therefore, the terms of "uni-part" and "bi-part" may be misleading. Sometimes, the parts in the so-called "uni-part characters" can even be the same as the parts in the so-called "bi-part characters" (e.g. 舍, in which both parts are frequently-used parts in the so-called "bi-part characters"). Second, it is definitely not the case that each radical in the so-called "bi-part characters" is a uni-part character, whereas it can be regarded as a uni-part character in this study. Many of them cannot be used independently. Moreover, many Chinese characters do have a "phonetic radical" which is in some way related to their pronunciation, these radicals, for various, especially, historical reasons, cannot be normally regarded as an exact and explicit marker of the characters' pronunciation. One assumption of this research is that the subjects can make ease use of the phonetic radicals to trigger the required pronunciation, but this point, for those characters that have come to be pronounced totally differently from their phonetic radicals (e.g.流); and for those which are pronounced only somewhat like their phonetic radicals (e.g.姿, which is used in this research), this point should be dubious.

It is accepted in general that scripts were invented to record verbal language. There have been developed two types of orthography and phonological one which may model two aspects of language as morphology and phonology. The match between script and phoneme could be invented in artificial rule, which is called as rebus but the connection between script and meaning is natural. For example, “羊” was invented to symbolized goat, then it was used to match pronunciation /yang/ of the word "ocean". This 
phonological matching is defined as rebus. So, /yang/ was used to match the two characters "goat" and "ocean" resulting in confusion of meaning. Overcoming this limitation, the meaning element of “沫” was attached to “羊” to make “洋”, which matches to the character "ocean" differentiating meaning from the character "ocean". This borrowing for phonology without any semantic as rebus was invented to develop the script from the simple pictorial sign (Jean 1992; Lee 2003) so that the script could represent phonology. Even if the definition of character pattern is somewhat particular, the phonological process still serves to make pronunciation with reservation of morphology.

This study manipulated uni-part and bi-part character patterns in different specific tasks to analyze the processes guided by semantic radicals and phonological radicals. Word or character frequency is regarded as a lexical variable. The lexical items could be searched by frequency; some items of high frequency might be faster and more accurate than others of low frequency when being processed. This study manipulated character frequency in a factorial design with other variables, to analyze lexical influences. This experiment analyzed four tasks; the lexical decision task, the meaning-matching task, the naming task and the pronunciation-matching task. The first two tasks are utilized to let the semantic routes govern response production, and the other two tasks activate the phonological route.

This study's results, with the statistical analysis, showed the significant interaction effect of the character patterns with the tasks, and the significant main effect of the frequency regardless of the tasks. The bi-part characters were processed slower than uni-part in the lexical decision task and the meaning-matching task. In contrast, with the other two tasks as with the naming task and the pronunciation-task, the bi-part characters were processed faster than uni-part. The above results were confirmed in replication with the same sort of tasks. The phonological radicals of the bi-part characters, and the differences of these radicals from the uni-part characters, contributed to inhibit meaning processes but facilitated phonological processes. The result suggests that the phonological radicals are activated to delay processes for meaning responses, and to help phonological processes. Even with the above explanation, in terms of the different sort of tasks it is a prerequisite that character activation with the phonological radicals is primary. The frequency effect was observed in each of the tasks, overall. This study has the important indications for the word recognition model, it suggests that the lexical route is activated not only in the meaning related tasks, but also the phonologically related tasks. Even though Chinese characters hold dominant semantic radicals, the phonological processes are inevitable. 


\section{Suggestion and conclusion}

The statistical data showed that the recognition of Chinese character could indeed be sensitive to the character patterns and word frequency conditions. Referring to the experimental data on the inhibition and facilitation of the phonological radical of bi-part character according to tasks, and the consistent frequency effect, phonological activation is primary, but lexical process is inevitable. Some studies concluded the primacy of phonological activation (e.g., Chen et al. 1988, Hoosain and Osgood 1983; Jin 2013; Hung and Tzeng 1981; Peng et al. 1985), and other studies remained to insist the lexical process is inevitable with Chinese (Baron and Strawson 1976; Leong 1986; Mattingly 1992). However, this study confirmed both the primacy of phonological process even in the semantically related tasks, and the inevitability of lexical process even in phonologically related tasks. The discussion succeeded to conceptualize a model, owing to which both processes were complimentary, not contradictory; the primacy of the phonological process is fast and precedent to other processes, the inevitability of lexical process is necessarily required over all tasks, even if it is delayed.

Further research needs to be conducted to examine this model in diverse orthographical systems such as Hangul (Korean), and others. Hangul orthography is not only conceptualized to be so transparent between letters ad phonemes, but also commented to sustain morphological syllable as a chunk. Lee (2003) concluded that the syllable is chunked to help the process for phonology and morphology, examining Hangul phonological rules that relied on the unit of the syllable chunk to suggest the phonological primacy processes (e.g., Lee et al. 2006). This suggestion also calls for further research to analyze the processing of syllables.

\section{References}

Baron, J., \& Strawson, C. 1976. Use of orthographic and word-specific knowledge in reading words aloud. Journal of Experimental Psychology: Human Perception and Performance, 2(3), 386-393.

Chen, M. J., Yung, Y. F., \& Ng, T. W. 1988. The effect of context on the perception of Chinese characters. In: I. M. Liu, H. C. Chen \& M. J. Chen (eds.), Cognitive aspects of the Chinese language (pp. 27-39). Hong Kong: Asian Research Service.

Coltheart, M. (1985). Cognitive neuropsychology and the study of reading. In: M. I. Posner., \& G. S. M. Matin (eds.) Attention and d performance XI (pp. 3 37). Hillsdale, N.J.: Erlbaum.

Coltheart, M., Curtis, B., Atkins, P., \& Hailer, M. 1993. Models of reading aloud: dual-route and parallel-distributed-processing approaches. Psychological review, 100(4), 589-608. 
Coltheart, M., Rastle, K., Perry, C., Langdon, R., \& Ziegler, J. 2001. DRC: A dual route cascaded model of visual word recognition and reading aloud. Psychological Review, 108, 204-256.

Coslett, H. B. 1991. Read but not write "idea": evidence for a third reading mechanism. Brain and Language, 40(4), 425-443.

Hoosain, R., \& Osgood, C. E. 1983. Information processing times for English and Chinese words. Perception and Psychophysics, 34, 573-577.

Hsieh. Shu-K. (Eds.) 2006. Hanzi, Concept and Computation: a preliminary survey of Chinese characters as a knowledge resource in Natural Language Processing. Tobias-lib: ub Tübingen, Germany.

Hung, D. L., \& Tzeng, O. J. L. 1981. Orthographic variations and visual information Processing. Psychological Bulletin, 90, 377-414.

Hung, D. L., Tzeng, O. J. L. \& Tzeng, A. K. Y. 1992. Automatic activation of linguistic information in Chinese character recognition. In: R. Frost \& L. Katz (eds.), Orthography, phonology, morphology, and meaning (pp. 119-130). Amsterdam: North-Holland.

Jean, G. (Eds.) 1992. The story of alphabets and scripts. London: Thames\& Hudson.

Jin Z. Nonselective access of English phonology in bi-scriptal Chinese-Korean visual word recognition. Cognitive Processing, 2013, 14, 435-441.

Lee, Y. 2003. The orthographical processes. In M. Jo (eds.), Psycholinguistics, edited (pp. 67-97). Seoul: Hakjisa.

Lee, Y., Moreno, A. M., Park, H., Carello, C., \& Turvey, M. T. 2006. Phonological assimilation \& visual word recognition. Journal of Psycholinguistic Research, 35(6), 513-530.

Leong, C. K. 1986. What does accessing a morphemic script tell us about reading and reading disorders in an alphabetic script? Annals of Dyslexia, 36, 82-102.

Levelt, W. J. M. 1999. Models of word production. Trends in Cognitive Science, 3(6), 223-232.

Lukatela, G., Frost, S. J., \& Turvey, M. T. 1999. Identity priming in English is compromised by phonological ambiguity. Journal of Experimental Psychology: Human Perception and Performance, 25, 775-790.

Mattingly, I. G. 1992. Linguistic awareness and orthographic form. In R. Frost \& L. Katz (Eds.), Orthography, phonology, molphology, and meaning (pp. 11-26). Amsterdam: Elsevier Science.

Neely, J. H. 1991. Semantic priming effects in visual word recognition: A selective review of current findings and theories. In D. Besner \& G. Humphreys (Eds.), Basic processes in reading: Visual word recognition (pp. 264-336). Hillsdale, NJ: Erlbaum.

Patterson, K. E., \& Morton, J. 1985. From orthography to phonology: an attempt at an old interpretation. In: K.E. Patterson, J. C. Marshall \& M. Coltheart, Surface dyslexia: neuropsychological and cognitive studies of phonological reading (pp. 335-359). Hillsdale, NJ: Erlbaum. 
Peng, D. L., Guo, D. J. \& Zhang, S. L. 1985. The retrieval of information of Chinese characters in making similarity judgment under recognition condition. Acta Psychologica Sinica, 17, 227-234.

Perfetti, C. A., Liu, Y. , \& Tan, L. H. 2002. How the mind can meet the brain in reading: A comparative writing system approach. In H. S. R. Kao, C.K. Leong, \& D. G. Gao (Eds.), Cognitive neuroscience studies of the Chinese language (pp. 36-60). Hong Kong: University Press.

Perfetti, C. A., Liu, Y., \& Tan, L. H. 2005. The Lexical Constituency Model: Some implications of research on Chinese for general theories of reading. Psychological Review, 112, 43-59.

Perfetti, C. A. \& Tan, L. H. 1998a. The time course of graphic, phonological, and semantic activation in visual Chinese character identification, Journal of Experimental Psychology: Learning, Memory, and Cognition 24: 101-118.

Perfetti, C. A. \& Tan, L. H. 1998b. The constituency model of Chinese character identification. In: J. Wang, A. Inhoff \& H.-C. Chen (eds.), Reading Chinese script: A cognitive analysis. Hillsdale, NJ: Erlbaum (in press).

Perfetti, C. A., \& Zhang, S. 1991. Phonemic processes in reading Chinese words, Journal of Experimental Psychology: Learning, Memory, and Cognition, 17, 633-643.

Perfetti, C. A., \& Zhang, S. 1995. The universal word identification reflex. The psychology of learning and motivation, Vol. 33 (pp. 159-189). San Diego, CA: Academic Press.

Seidenberg, M. S., \& McClelland, J. L. 1989. The distributed development model of word recognition and naming. Psychol. Rev, 96, 523-568.

Strain, E., Patterson, K. E., \& Seidenberg, M. S. 1995. Semantic influence on word recognition. Journal of Experimental Psychology: leaning, memory, and cognition, 21, 1140-1154.

Tan, L. H., \& Perfetti, C. A. 1997a. Visual Chinese character recognition: Does phonological information mediate access to meaning?, Journal of Memory and Language 37: 41-57.

Tan, L. H., \& Perfetti, C. A. 1998. Phonological codes as early sources of constraint in Chinese word identification: A review of current discoveries and theoretical accounts. Reading and Writing, 10, 165-200.

Tan, L. H., Hoosain, R. \& Siok,W.W. T. 1996. The activation of phonological codes before access to character meaning in written Chinese, Journal of Experimental Psychology: Learning, Memory, and Cognition 22: 865-883.

Van Orden, G. C., \& Goldinger, S. D. 1994. Interdependence of form and function in cognitive systems explains perception of printed words. Journal of Experimental Psychology: Human Perception and Performance, 20, 1269-1291. 
Wang,Q., Chi,Z.R., Feng,D.D., \& Zhao,R.C. 2000. "Hidden Markov random field based approach for off-line handwritten Chinese character recognition," Accepted by the $15^{\text {th }}$ Int. Conf. on Pattern Recognition, Barcelona, Spain.

$\mathrm{Xu}$, Y., Pollatsek, A., \& Potter, M. 1999. The activation of phonology during silent Chinese word reading. Journal of Experimental Psychology: Learning, Memory, and Cognition, 25, 838-857. 